\title{
Uma visão geral de um país em desenvolvimento sobre homens vítimas de violência física e sexual
}

RESUMO | Objetivo: Analisar os dados de vitimização de homens, com idade entre 20 e 59 anos, pelas distintas formas de violência física e sexual, registrados no Sistema de Vigilância de Violências e Acidentes/SIVVA da cidade de São Paulo (SP). Método: Trata-se de um estudo transversal, de abordagem quantitativa, descritivo, retrospectivo. Realizado por meio das notificações do Sistema de Informação para a Vigilância de Violência e Acidentes (SIVVA) da Secretaria Municipal de Saúde da cidade de São Paulo, no período de janeiro a dezembro de 2014 na cidade de São Paulo, sendo composto por 6658 homens na faixa etária entre 20 a 59 anos. Resultados: O número de casos de violência física foram 6.624 casos e violência sexual 34 casos contra homens com idade entre 20 e 59 anos. O vínculo do agressor em relação a vítima era familiar ou conhecido da vítima; $61,8 \%$ violência sexual e $42,8 \%$ violência física. Conclusão: O estudo evidenciou que as vítimas eram predominantemente adultos jovens. A violência na maioria dos estudos é vista como um grande problema social que atinge toda a sociedade, sendo responsável pelo adoecimento e mortes causadas por ações realizadas por indivíduos ou, provocando danos físicos, emocionais as vítimas; além de gerar despesas para os serviços público.

Palavras-chaves: Violência Sexual; Saúde do Homem; Educação Sexual; Saúde Pública.

ABSTRACT | Objective: the analyze the victimization data of men, aged between 20 and 59 years, by the different forms of physical and sexual violence, registered in the Violence and Accident Surveillance System / SIVVA in the city of São Paulo (SP). Method: This is a cross-sectional study, with a quantitative, descriptive, retrospective approach. Carried out through the notifications of the Information System for the Surveillance of Violence and Accidents (SIVVA) of the Municipal Health Department of the city of São Paulo, in the period from January to December 2014 in the city of São Paulo, consisting of 6658 men aged between 20 and 59 years. Results: The number of cases of physical violence was 6,624 cases and sexual violence 34 cases against men aged between 20 and 59 years. The aggressor's relationship with the victim was familiar or known to the victim; $61.8 \%$ of sexual violence and $42.8 \%$ of physical violence. Conclusion: The study showed that the victims were predominantly young adults. Violence in most studies is seen as a major social problem that affects the whole of society, being responsible for illness and deaths caused by actions performed by individuals or, causing physical harm, emotional victims; in addition to generating expenditure for public services

Keywords: Sexual Violence; Men's Health; Sex Education; Public Health.

RESUMEN | Objetivo: Analizar los datos de victimización de hombres, de entre 20 y 59 años, debido a las diferentes formas de violencia física y sexual, inscritas en el Sistema de Vigilancia de La Violencia y Accidentes/SIVVA de la ciudad de Sao Paulo (SP). Método: Se trata de un estudio transversal, con un enfoque cuantitativo, descriptivo y retrospectivo. Realizado a través de las notificaciones del Sistema de Información para la Vigilancia de la Violencia y accidentes (SIVVA) del Departamento Municipal de Salud de la ciudad de Sao Paulo, en el período de enero a diciembre de 2014 en la ciudad de Sao Paulo, con una crianza de 6658 hombres de entre 20 y 59 años. Resultados: El número de casos de violencia física fue de 6.624 casos y violencia sexual 34 casos contra hombres de entre 20 y 59 años. El vínculo del agresor con la víctima era familiar o conocido por la víctima; 61,8\% de violencia sexual y $42,8 \%$ de violencia física. Conclusión: El estudio mostró que las víctimas eran predominantemente adultos jóvenes. La violencia en la mayoría de los estudios es vista como un problema social importante que afecta a toda la sociedad, siendo responsable de enfermedades y muertes causadas por acciones realizadas por individuos o, causando daño físico, víctimas emocionales; además de generar gastos para los servicios públicos.

Palavras claves: Violencia Sexual; Salud de los Hombres; Educación Sexual; Salud Pública.

\section{Jane de Eston Armond}

Médica, Doutora em Saúde Pública da Faculdade de Saúde Pública- USP, Docente do Stricto Sensu Ciências da Saúde da Universidade Santo Amaro.

ORCID: 0000-0003-1561-8113

\section{Rodrigo de Eston Armond}

Fisioterapeuta, Mestrando do programa Ciências da Saúde da Universidade Santo Amaro.

ORCID: 0000-0001-7371-1354

\section{Caio Vinicius Fonseca da Silva}

Discente do Curso de Medicina da Universidade Santo Amaro.

ORCID: 0000-0001-7520-8744

\section{Cintia Leci Rodrigues}

Biomédica, Mestre em Saúde Pública da Faculdade de Saúde Pública- USP, Docente do Curso de Medicina da Universidade Santo Amaro. ORCID: 0000-0001-8064-2203

\section{Jefferson Carlos de Oliveira}

Enfermeiro, Mestrado Profissional em Enfermagem pelo Processo de Cuidar em Saúde do Centro Universitário São Camilo, Docente do Curso de Enfermagem e Pós-Graduação em Urgência e Emergência- Centro Universitário Anhanguera de São Paulo- Vila Mariana. ORCID: 0000-0002-5258-7099

Recebido em: 19/05/2020

Aprovado em: 27/08/2020
INTRODUÇÃO

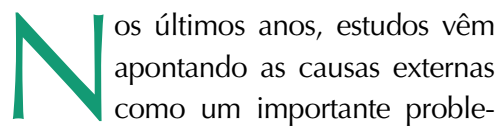
ma de saúde pública, representando importante causa de morbidade e mortalidade na população mundial e brasileira, especialmente entre a população do sexo masculino $^{1,2}$. A temática sobre a situação de saúde do homem tem alcançado destaque no Brasil, principalmente devido aos elevados índices de morbidade e mortalidade decorrentes de causas externas que afetam 
esse grupo, bem como a sua baixa procura pelos serviços de atenção primária à saúde, que poderia minimizar esse agravante. Os indicadores de morbidade relacionados às causas externas podem oferecer uma visão ampliada do processo de vulnerabilidade a agravos à saúde da população masculina, com o objetivo de refletir sobre o comportamento e vida social dessa população, assim como identificar dados epidemiológicos que ilustrem os problemas mais prementes em sua saúde ${ }^{2}$.

Entre as principais causas de morbimortalidade vem se destacando a violên$\mathrm{cia}^{3}$. Estas por sua vez, são reconhecidas como violação dos direitos humanos e um grave problema de saúde por suas complicações para suas implicações para a saúde física e emocional das vítimas, como consequências negativas para o desenvolvimento afetivo e social, pela possibilidade de replicação do fenômeno em futuras gerações ${ }^{3}$.

Uma abordagem intersetorial é necessária para a compreensão sobre o tema violência, que envolve fatores de segurança pública, de justiça, de educação e de saúde. O papel atual da saúde sobre esse agravo, não deve restringir-se à cura e à reabilitação, mas deve abarcar estratégias de prevenção e de promoção ${ }^{4}$.

Apesar das severas consequências decorrentes da violência em ambos os sexos, a maioria das pesquisas que inves-

\section{Figura 1. Tipo de violência (física e sexual) acometida contra homens, segundo a faixa etária, São Paulo, ano 2014.}

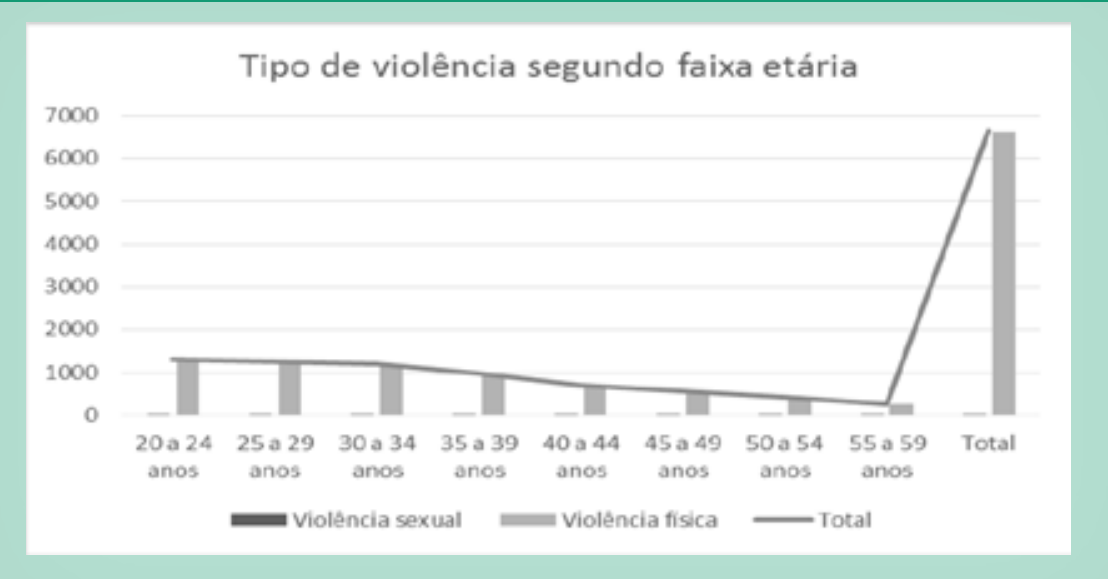

Fonte: Secretaria Municipal de Saúde da cidade de São Paulo, 2020.

tigam a violência é voltada às mulheres, crianças e adolescentes e idosos como vítimas e aos homens como agressores. Há escassez de estudos que contemplem a questão da violência física e violência sexual em homens ${ }^{5}$. Este artigo tem como objetivo analisar os dados de vitimização de homens, com idade entre 20 e 59 anos, pelas distintas formas de violência física e sexual, registrados no Sistema de Vigilância de Violências e Acidentes/SIVVA da cidade de São Paulo (SP).

\section{MÉTODO}

Trata-se de um estudo transversal, de abordagem quantitativa, descritivo, retrospectivo. O estudo abrangeu a totalidade de 6658 homens com idade entre 20 e 59 anos, vítimas de violência física e sexual, notificadas no Sistema de Informação para a Vigilância de Violência e Acidentes (SIVVA) da Secretaria Municipal de Saúde da cidade de São Paulo, onde são registradas as notificações de acidentes e violência por meio da ficha de notificação de casos suspeitos ou confirmados6, no período de janeiro a dezembro de 2014 na cidade de São Paulo.

As variáveis utilizadas para o estudo foram: idade, sexo masculino, tipo de violência (física e sexual), frequência da violência, vinculo do agressor em relação à vítima (familiar e outros conhecidos, des- 
Conforme mostrado na figura 1 , a violência física e sexual foram mais frequentes nos adultos jovens. No que tange a violência sexual, $73,5 \%$ ocorreram na faixa etária de 20 a 39 anos de idade (adultos jovens). No que concerne à violência física $71,1 \%$ nos adultos jovens.

Em relação a frequência da agressão, na violência física: 41,6\% primeira vez da agressão e 11,2\% sofreram violência física mais de uma vez. Na violência sexual: $32,4 \%$ primeira vez e $23,5 \%$ mais de uma vez que sofreram violência sexual.

O vínculo do agressor em relação a vítima era familiar ou conhecidos da vítima; $61,8 \%$ violência sexual e 42,8\% violência física. Vale ressaltar que na violência física o vínculo do agressor com a vítima, 8,7\% eram ladrões ou assaltantes, $17,4 \%$ outros desconhecidos e $0,5 \%$ patrão ou chefia da vítima.

Segundo dados acerca do sexo do agressor, houve uma predominância do sexo masculino; na violência física $(17,4 \%)$ e na violência sexual $(32,3 \%)$. Os dados referentes a faixa etária do agressor, tanto na violência física quanto na violência sexual eram adultos jovens.

O principal instrumento de agressão na violência física contra homens foi o uso da força corporal (63,0\%), arma branca $(9,8 \%)$, objeto contundente $(8,0 \%)$, arma de fogo $(4,7 \%)$. No que tan- ge a violência sexual: uso da força corporal 44,1\%, arma branca 5,9\%.

Dados notificados sobre o diagnóstico de lesão em vítimas de violência física foram: traumatismos da cabeça $(41,2 \%)$, traumatismos envolvendo múltiplas regiões do corpo $(19,6 \%)$, traumatismos de região não especificada do tronco, membro ou outra região do corpo $(8,4 \%)$. Os dados notificados em relação ao diagnóstico de lesão entre as vítimas de violência sexual foram, respectivamente, abuso sexual (26,5\%), abuso psicológico (5,9\%), os demais foram dados ignorados.

A evolução dos casos dos atendimentos às vítimas de violência física e sexual, 70\% teve alta hospitalar imediata. $\mathrm{Na}$ violência física, 65 casos foram a óbito durante o atendimento ou foram recebidos em óbito no serviço de saúde.

Sobre o local da ocorrência da violência (física e sexual), 18,5\% ocorreram na residência da vítima e $17,9 \%$ em via pública. O horário da ocorrência durante a noite e madrugada. Dos casos notificados de violência física e sexual contra homens, 11 casos destas vítimas tinham deficiência física e 13 casos de deficiência mental.

\section{DISCUSSÃO}

A violência é um fenômeno comple-

Figura 2. Instrumento de agressão utilizado contra homens vítimas de violência física na cidade de São Paulo, 2014.

\section{Violência Física segundo instrumento da agressão}

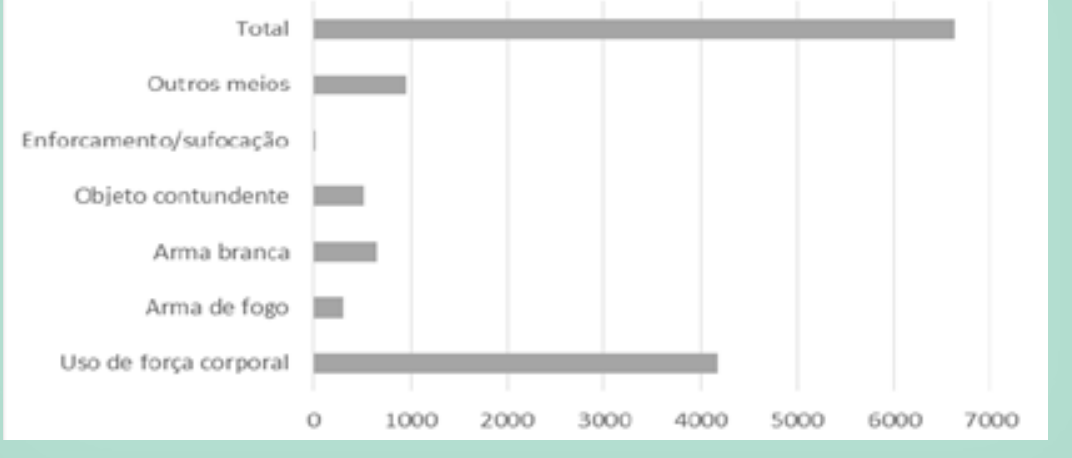

Fonte: Secretaria Municipal de Saúde da cidade de São Paulo, 2020. xo e uma questão de saúde pública ${ }^{7}$. No presente estudo foram notificados 6.658 casos de violência física e sexual contra homens, sendo estas vítimas predominantemente adultos jovens.

A violência na maioria dos estudos é vista como um grande problema social que atinge toda a sociedade, prejudicando crianças, adolescentes, mulheres, homens e idosos; sendo responsável pelo adoecimento e mortes causadas por ações realizadas por indivíduos ou, provocando danos físicos, emocionais as vítimas; além de gerar despesas para os serviços públicos ${ }^{8}$.

Alguns estudos apontaram que existem em muitos atendimentos a subnotificação, pela falta de monitoramento e de orientação para um registro contínuo, padronizado e adequado sobre a violência8.

Garbin e cols (2015) elucida que esta situação da subnotificação se repete quando as vítimas da violência são crianças, mulheres, homossexuais, idosos, doentes, pobres e moradores de rua, o que leva a interpretar que existem pessoas não reconhecidas como cidadãos e que carecem de direitos ${ }^{8}$.

Neste estudo foram descritos os casos notificados de violência física e sexual contra homens, no que tange a violência sexual, foram notificados 34 casos, além de a literatura apontar a subnotificação, quando se trata de estudar o sexo masculino, reflete a baixa procura deste grupo populacional aos serviços de saúde, explicada por questões culturais relacionadas ao machismo. Em geral, os homens acreditam que as mulheres necessitam mais cuidados, enquanto eles devem manter uma postura de força viril, sem demonstrar qualquer sinal de fraqueza, medo, ansiedade ou insegurança ${ }^{9}$.

Em relação à frequência da agressão, na violência física: $41,6 \%$ e 32,4\% na violência sexual foram notificados como a primeira vez da violência, uma limitação deste estudo é não poder concluir se foi a primeira vez que estes homens adultos jovens foram vítimas de violência ou se foi a primeira vez notificada no serviço de 
saúde. A conscientização da importância da notificação, a quebra de paradigmas e o treinamento contínuo no diagnóstico de situações de violência, trazem subsídios para a construção de políticas públicas em saúde mais eficazes, vigilância sentinela dos casos de violência, assim contribuindo para a solução de um expressivo problema da sociedade ${ }^{8}$.

Segundo o local da violência, neste estudo a residência foi o principal local e o vinculo do agressor com a vítima sendo familiares ou conhecidos.

Outro ponto marcante observado neste estudo é a ênfase dada por outros autores com o foco no homem como agressor e não como vítima. Apesar dos agressores serem conhecidos e/ou familiares, predominantemente do sexo masculino e adultos jovens, uma limitação deste estudo foi não poder identificar a relação conjugal. Segundo Cezario e cols (2015), com o foco no homem como vítima em relacionamentos heterossexuais, e da violência entre parceiros íntimos em relacionamentos homoafetivos, percebe-se que o número de publicações sobre a temática ainda é pequeno e pouco discutido no universo científico, se comparado à violência contra a mulher. Dentro da violência entre parceiros íntimos, tanto em relacionamentos homossexuais quanto em relacionamentos heterossexuais, a mulher ainda aparece como principal vítima, mesmo quando o homem também é citado ${ }^{10}$.

Quanto ao tipo do instrumento de agressão, destaca-se o uso da força corporal empregada através de tapas, chutes e soco. Assim como encontrado em outros estudos o uso da força corporal contra muIheres, idosos e crianças e adolescentes vitimados. Na literatura científica é escasso estudos com homens vitimados ${ }^{11}$.

A necessidade de cuidados de saúde após a agressão sugere o quanto à violência gera impacto na saúde, podendo trazer graves consequências, tanto no âmbito físico quanto mental. Uma possível consequência dos homens não procurarem os serviços de saúde, a sub- notificação dos casos de violência pode se justificar pela percepção da violência como problema policial e não como um problema de saúde ${ }^{11}$.
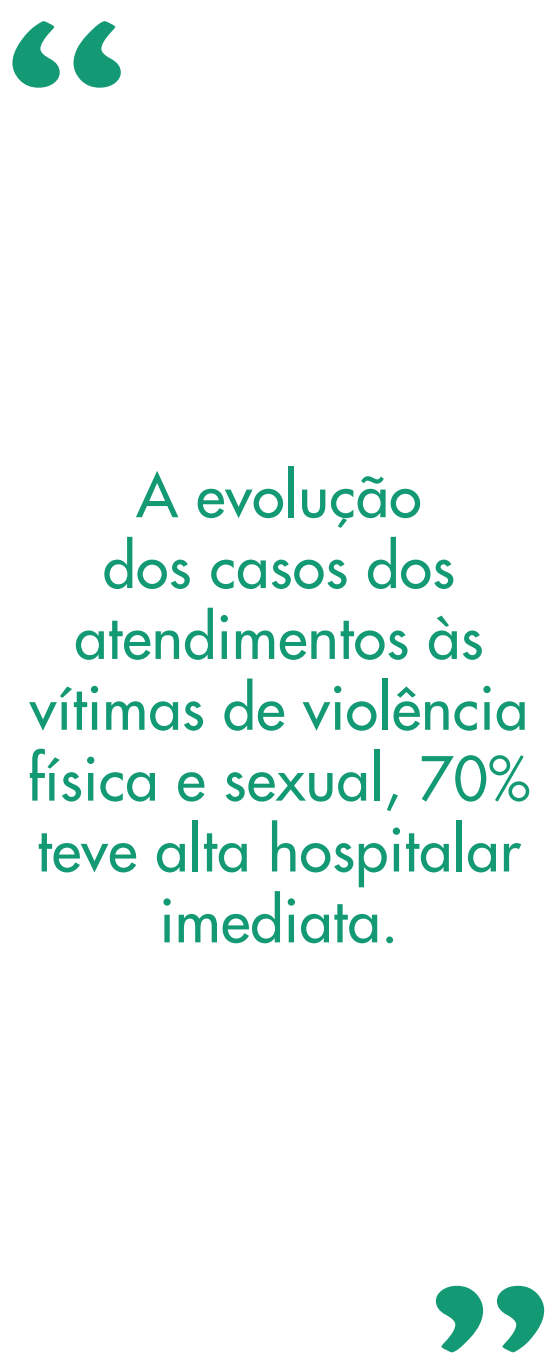

Os episódios violentos envolvendo o uso de arma branca e arma de fogo, no presente estudo foi o instrumento utilizado tanto na violência física e sexual. Uma vez que os atos violentos praticados com arma de fogo provocam mais vítimas fatais, tendo maior impacto nas estatísticas de mortalidade. No presente trabalho a maioria das vítimas de agressões e encontrava-se em faixa etária economicamente produtiva, entre 20 a 39 anos. O fato de a violência incidir predominantemente sobre vítimas jovens é preocupante visto que determina altas taxas de anos potenciais de vida perdidos (APVP), privando a sociedade da contribuição econômica e social. As vítimas de arma branca, ao contrário, sobrevivem em sua maioria, e demandam tratamento médico, sendo incorporadas nas estatísticas de morbidade ${ }^{12}$.

A respeito das lesões e locais atingidos, confirma-se a informação que a região da cabeça é o mais prevalente, sendo o diagnóstico de lesão traumatismos da cabeça $^{13}$. Estes dados corroboram com a literatura, onde a cabeça e face foram as regiões do corpo mais atingida por vítimas de violência, em relatos clínicos foram as contusões, lacerações e fraturas $^{14,15,16}$.

A evolução dos casos dos atendimentos às vítimas de violência física e sexual, $70 \%$ teve alta hospitalar imediata. Isto reflete em conhecer as lesões de menor gravidade, as quais não levam a óbito ou internação, mas são responsáveis por demanda nos serviços de saúde e podem resultar em danos à saúde física e mental para os homens. Para isto, é necessário o planejamento de políticas públicas, buscando maior efetividade da atenção à saúde do homem ${ }^{17}$.

O envolvimento masculino em maior proporção, com violência física e sexual, segundo o local da ocorrência, sendo a residência, seguido por via pública. É apontado por alguns autores como uma questão cultural e de gênero, uma vez que a violência constitui uma forma de resolução de conflitos entre os homens, comportamento aceitável socialmente desde a existência humana. A idade caracterizada por adultos jovens, uso da força física, o uso da arma branca e o uso da arma de fogo são resultados que coincidem com outras pesquisas e que refletem o comportamento desafiador prevalente entre diferentes culturas, em que participar de brigas e portar armas constitui uma forma comum de violência interpessoal no sexo masculino. Alguns estudos ainda apontam este comportamento como resultante de fatores familiares e sociais, entre outros ${ }^{18}$. 
O período de a ocorrência ser preferencialmente durante a noite e a madrugada sugere-se onde homem está em sua residência após o seu dia de trabalho e em via pública podendo estar na volta de sua atividade laboral ou em horário de lazer. Outro dado também referendado na literatura como uma prática recorrente, facilitada pelo fato de a agressão ocorrer sem interrupções de outras pessoas, sob a privacidade do lar e no período noturno. Este ambiente doméstico propicia que familiares ou pessoas conhecidas e de confiança sejam os agressores, tornando a violência mais fácil de ser realizada e dificultando sua identificação ${ }^{14}$.

$\mathrm{Na}$ presente casuística, homens vitimados por agressão física e/ou sexual, 24 casos possuía algum tipo de deficiência, seja física ou mental. Vale ressaltar a provável subnotificação das agressões e o preenchimento inadequado das fichas de notificação de casos suspeitos e/ou confirmados de acidentes e violência pelos profissionais de saúde ${ }^{19}$. São discutidos como possíveis contribuintes para geração de conflitos, tendo por resultado o comprometimento e a desestruturação familiar. No exposto, acredita-se que a violência é instalada, vitimizando em especial os homens com deficiência, por se constituírem um público de vulnerabilidade ${ }^{20}$.

Vale ressaltar, a importância dos estudos gênero no âmbito da Saúde Coletiva, uma vez que a masculinidade tem se configurado como fator relevante na relação entre indivíduos e serviços de saúde; é imperativo o desenvolvimento de tecnologias capazes de envolver e responsabilizar o homem por sua saúde, além de sensibilizá-lo diante da necessidade de ações de prevenção e promoção de saúde. E mesmo já tendo sido demonstrado que as taxas de morbimortalidade masculinas são maiores para quase todas as causas, devido à baixa procura dos homens pelos serviços de saúde ${ }^{21}$.

\section{CONCLUSÃO}

A característica da violência física e sexual entre homens mostraram que as maiores vítimas são adultos jovens (20 a 39 anos de idade), os agressores do sexo masculino e por uso da força corporal, o que pode resulta em impacto negativo na força produtiva do país, além de onerar os gastos do setor saúde, como por exemplo, a recuperação e a reabilitação das vítimas, afetando a produtividade social e econômica.

Tornam-se essenciais ações de prevenção e atendimento em níveis individual, familiar, social e cultural para que se possa reverter a realidade identificada neste trabalho.

\section{References}

1. Tavares FL, Leite FMC, Lima EFA, Cade NV, Coelho MJ. Homens e acidentes motociclístico: gravidade dos acidentados a partir do atendimento pré-hospitalar. J. res. fundam. care. Online. 2016; 8 (1): 4004-4014.

2. Santos VC, Santos MG, Vilela ABA, Nery AA, Casotti CA, Boery EN. Padrões de mudança na saúde do homem a partir de indicadores demográficos e epidemiológicos. J. res. fundam. care. Online. 2015; 7 (2): 2569-2581.

3. Lima JS, Deslandes SF. Olhar da gestão sobre a implantação da ficha de notificação da violência doméstica, sexual e/outras violências em uma metrópole do Brasil. Saude Soc. 2015; 24 (2): 661-673.

4. Garcia Filho C, Sampaio JJC. Interfaces entre a história da violência e a constituição do território no Ceará: um esforço de síntese e periodização. Saude Soc. 2014; 23 (4): 1209-1221.

5. Lindner SR, Coelho EBS, Bolsoni CC, Rojas PF, Boing AF. Prevalência de violência física por parceiro íntimo em homens e mulheres de Florianópolis, Santa Catarina, Brasil: estudo de base populacional. Cad. Saúde Pública. 2015; 31 (4): 815-826.

6. São Paulo. Secretaria Municipal de Saúde. Sistema de Informação e Vigilância de Violências e Acidentes - SIVVA [Internet]. São Paulo: Prefeitura de São Paulo; 2013 [acesso em 16 março. 2016]. Disponível em: http:// www.prefeitura.sp.gov.br. 7. Rosa LW, Falcke D. Violência conjugal: compreendendo o fenômeno. Rev. SPAGESP. 2014; 15 (1): 17-32.

8. Garbin CAS, Dias IA, Rovida TAS, Garbin AJI. Desafıos do profıssional de saúde na notifıcação da violência: obrigatoriedade, efetivação e encaminhamento. Ciênc saúde coletiva. 2015; 20 (6): 1879-1890.

9. Codogno JS, Turi BC, Fernandes RA, Monteiro HL. Comparação de gastos com serviços de atenção básica à saúde de homens e mulheres em Bauru, São Paulo, 2010. Epidemiol. Serv. Saúde. 2015; 24 (1): 115-122.

10. Cezario ACF, Fonseca DS, Lopes NC, Lourenço LM. Violência entre parceiros íntimos: uma comparação dos índices em relacionamentos hetero e homossexuais. Temas psicol. 2015; 23 (3): 565-575.

11. Leite FMC, Bravim LR, Lima EFA, Primo CC. Violência contra a mulher: caracterizando a vítima, a agressão e 0 autor. J. res. fundam. care. online.
2015; 7 (1): 2181-2191.

12. Ferreia TFA, Ribeiro LA. Epidemiologia das agressões atendidas pelo hospital das clínicas de Uberlândia e dos homicídios ocorridos no município de 2000 a 2004. Biosci J. 2009; 25 (2): 161-170.

13. Garbin CAS, Queiroz APDG, Rovida TAS, Garbin AJl. Divergências entre histórico da consulta e diagnóstico médico de agressões físicas registradas nos prontuários de uma unidade de urgência e emergência. Physis. 2013; 23 (3): 951-964.

14. Figueiredo MC, Cesar MO, Silva JP, Borba EMB. Prevalência de mulheres vítimas de violência no município de Porto Alegre e a influência de suas variáveis no âmbito odontológico. RFO. 2012; 17 (3): 254-260.

15. Souza RB, Todeschini AB, Veiga JCE, Saade N, Aguiar GB. Traumatismo cranioencefálico por projétil de arma de fogo: experiência de 16 anos do serviço de neurocirurgia da Santa Casa de São Paulo. Rev. Col. Bras. Cir. 2013; 40 (4): 300-304.

16. Correia TMP, Leal MCC, Marques APO, Salgado RAG, Melo HMA. Perfil dos idosos em situação de violência atendidos em serviço de emergência em Recife -PE. Rev. bras. geriatr. gerontol. 2012; 15 (3): 529-536.

17. Malta DC, et al. Causas externas em adolescentes: atendimentos em serviços sentinelas de urgência e emergência nas Capitais Brasileiras - 2009. Ciênc. Saúde Coletiva. 2012; 17 (9): 2291-2304.

18. Martins CBG, Mello Jorge MHP. Óbitos por causas externas em Cuiabá, 0 a 24 anos: perfil das vítimas e famílias segundo a intencionalidade. Rev. bras. epidemiol. 2013; 16 (2): 454-468.

19. Rodrigues CL, Armond JE, Górios C. Agressões físicas e sexuais contra idosos notificadas na cidade de São Paulo. Rev. Bras. Geriatr. Gerontol. 2015; 18 (4): 755-760.

20. Lima CC, Santos LES. Crianças vítimas de violência e autores da violência. Pediatr. mod. 2014; 50 (4).

21. Bispo $A$, Dias $A B$, Pereira $A$. Procura por cuidados de saúde: questões de gênero e raça entre colaboradores negros de uma universidade. J. res. fundam. care. online. 2015; 7 (1): 1856-1866. 